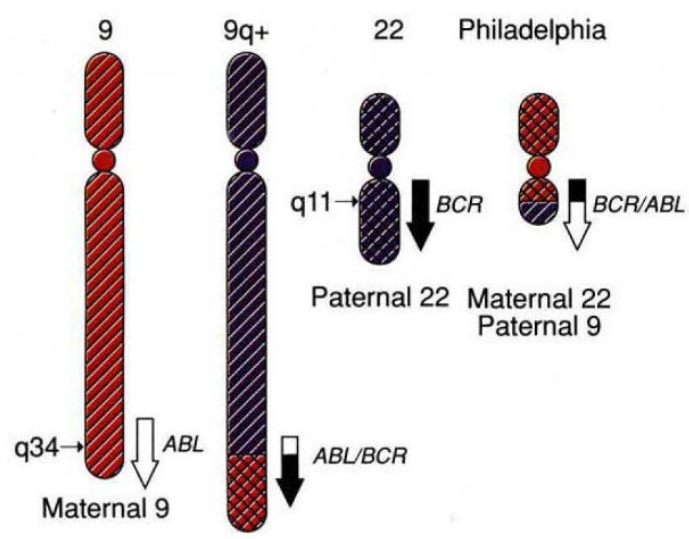

Paternal 9

Maternal 22

Parental origin of chromosomes involved in the Philadelphia translocation. The typical karyotype of peripheral white blood cells from patients with chronic myeloid leukaemia is shown. Chromosomes 9 and 22 are translocated so that a normal chromosome 9 is present in addition to a $9 \mathrm{q}^{+}$with some material from 22 attached to the long arm, and a normal 22 is seen together with the Philadelphia chromosome (22 with a bit of 9 attached). The translocation breakpoints are at $9 q 34$ and at 22q11 and lead to the formation of a fusion gene between $A B L$ and $B C R$ on $9 \mathrm{q}^{+}$and between $B C R$ and $A B L$ on the Philadelphia chromosome. The arrows indicate transcriptional orientation of the two genes. The study by Haas et al. shows that most translocated chromosomes 9 are paternal in origin (blue), whereas translocated chromosomes 22 are maternal (red).

some. This would lead to expression of $A B L / B C R$ whenever the paternal 9 is translocated, in turn suggesting that both the $\mathrm{BCR} / \mathrm{ABL}$ and the $\mathrm{ABL} / \mathrm{BCR}$ products are necessary for leukaemia to occur. But this possibility seems to be at odds with gene-transfer studies in the mouse, in which $B C R / A B L$ alone can induce leukaemic disease.

Could a look at the mouse be helpful, assuming that imprinting is conserved? The mouse $a b l$ gene is located on proximal chromosome 2, whereas bcr is on the middle part of chromosome 10 . The $a b l$ gene, therefore, is in a region that is known to be imprinted (by genetic criteria), whereas the $b c r$ region is not. Maternal disomy of proximal chromosome 2 leads to early embryonic death ${ }^{7}$, and if the $a b l$ gene were responsible for this phenotype it should also be observed in mice with (null) mutations of their $a b l$ gene. Fortunately, the experiment has already been done (by homologous recombination), but the outcome is quite different from the prediction: mice without functional $a b l$ genes develop happily to term, whereupon they show reduced viability ${ }^{8,9}$. An effect of parental transmission of the mutant alleles has not been reported.

If $A B L$ and $B C R$ were reciprocally imprinted, we should expect to see this simply by looking at transcription of the four different RNAs in leukaemic cells of Philadelphia-positive patients. We NATURE · VOL 359 - 1 OCTOBER 1992 should expect to see the $B C R /$ $A B L$ transcript but not the $B C R$ transcript, and the $A B L / B C R$ but not the $A B L$ transcript. Again we draw a blank leukaemic cells as well as leukaemic cell lines usually express messages for $B C R, A B L$ and $B C R / A B L$. (Again, I find it curious that mention of $A B L /$ $B C R$ is not to be found in the literature, although presumably this fusion transcript could be of a size similar to $B C R / A B L$.) Imprinting of these genes, therefore, if present in some progenitor cell population, is not maintained in leukaemic cells that appear in the periphery. On the whole, however, it seems that there is little evidence in either man or mouse for imprinting of $A B L$ or $B C R$.

There is another puzzling feature of transcription of $B C R$ and $B C R / A B L$. When individual Philadelphia-positive haematopoietic colonies were analysed for expression, they were found to transcribe either the $B C R$ or the $B C R / A B L$ message, but not both at the same time ${ }^{10}$. Might random dosage compensation (as in X-chromosome inactivation) operate at a stage where parental imprints are no longer in place? Finally, if this were not enough speculation, it is possible that position effects are exerted by the translocation breakpoints on other imprinted genes on chromosomes 9 and 22. This could result in either an increased or a decreased dosage of gene products that may contribute to the proliferative potential of myeloid lineages.

The study by Haas et al. raises a number of fascinating questions, to none of which there is a simple answer at present. The Philadelphia chromosome still holds a secret or two.

Wolf Reik is a Fellow of the Lister Institute of Preventive Medicine and is in the Department of Molecular Embryology, Institute of Animal Physiology and Genetics Research, Babraham, Cambridge CB2 4AT, UK.

1. Haas, O. A., Argyriou-Tirita, A. \& Lion, T. Nature 359 414-416 (1992).

2. Daley, G. Q. \& Ben-Neriah, Y. Adv. Cancer Res. 57. 151-184 (1991).

3. Campbell, M. L. \& Arlinghaus, R. B. Adv. Cancer Res. 57, 227-256 (1991)

4. Pendergast, A. M., Muller, A. J., Havlik, M. H., Maru, Y \& Witte, O.N. Cell 66, 161-172 (1991)

5. Diekmann, D. et al. Nature 351, $400-402$ (1991).

6. Surani, M. A. \& Reik, W. (eds) Genomic Imprinting in Mouse and Man, Sem. dev. Biol. Vol. 3 (Saunders Mouse and Man, Sem. dev.
Scientific, London, 1992).

7. Cattanach, B. M. \& Beechey, C. V. Developrnent (suppl.) 63-72 (1990).

8. Tybulewicz, V. L. J. et al. Cell 65, 1153-1164 (1991) 9. Schwartzberg, P. L. et al. Cell 65, 1165-1176 (1991)

10. Keating, A. \& Wang, X. H. Exp. Haematol. 19, 313 (1991).

\section{Relative differences}

INTUITION if nothing else would tell one that moas and kiwis, both flightless birds (ratites) and both inhabitants of New Zealand, are especially close relatives. Not so, say A. Cooper et al. (Proc. natn. Acad. Sci. U.S.A. 89, 8741$8744 ; 1992)$. Cooper and colleagues applied sequence and phylogenetic analysis to part of the mitochondrial $12 \mathrm{~S}$ ribosomal RNA gene of various ratites; moas are extinct, but the authors were able to extract usable samples from the tissue and bone of museum specimens. They find that moas diverged fairly early on in ratite evolution. Kiwis, by contrast, had a more recent ancestor in common with the emu, cassowaries and the ostrich. So New Zealand may well have been colonized independently by moa and kiwi forebears - with the possibility that the ancestors of kiwis arrived later by air, only then adapting to a grounddwelling life.

\section{Acid test}

PHOTOCHEMICAL reactions in cloud water droplets may be a significant source of organic peroxides and 'singlet' (excitedstate) molecular oxygen, all key players in atmospheric chemistry (B. C. Faust and J. M. Allen J. geophys. Res. 97, $12,913-12,926 ; 1992)$. The authors borrowed droplet samples collected by colleagues and irradiated them with simulated and natural sunlight. Although the resulting concentrations of oxidants seem small, a few nanomolar for the peroxides and less for the singlet oxygen under 'midday' conditions, they compete in importance with those generated by absorption into the droplets of the species created in air, previously thought to be the only process involved. The reaction mechanisms involved are not known. But given that peroxides and singlet oxygen are leading species responsible for oxidizing sulphur dioxide to sulphuric acid and for scavenging tropospheric ozone, the process clearly warrants further study.

\section{Smoke screen}

A CAUTIONARY tale for the common man is told by G. D. Smith and colleagues, who after digging into a huge existing dataset have unearthed an association between smoking and suicide (Lancet 340, 709-712; 1992). But this finding is one with a difference: although the association is strong, shows a relationship between dose and response, and seems clear of confounding factors, the authors simply don't believe it (or at least don't consider that here is a case of cause and effect). Their point is that epidemiologists are continually coming up with such associations, most of which may seem much more biologically plausible than their example but which may be equally spurious. 